\title{
Resolutions of operads via Koszul (bi)algebras
}

\section{Pedro Tamaroff ${ }^{1}$}

Received: 14 March 2021 / Accepted: 10 February 2022 / Published online: 3 March 2022

(c) The Author(s) 2022

\begin{abstract}
We introduce a construction that produces from each bialgebra $H$ an operad Ass $_{H}$ controlling associative algebras in the monoidal category of $H$-modules or, briefly, $H$-algebras. When the underlying algebra of this bialgebra is Koszul, we give explicit formulas for the minimal model of this operad depending only on the coproduct of $H$ and the Koszul model of $H$. This operad is seldom quadratic - and hence does not fall within the reach of Koszul duality theory-so our work provides a new rich family of examples where an explicit minimal model of an operad can be obtained. As an application, we observe that if we take $H$ to be the mod-2 Steenrod algebra $\mathcal{A}$, then this notion of an associative $H$-algebra coincides with the usual notion of an $\mathcal{A}$-algebra considered by homotopy theorists. This makes available to us an operad $\operatorname{Ass}_{\mathcal{A}}$ along with its minimal model that controls the category of associative $\mathcal{A}$-algebras, and the notion of strong homotopy associative $\mathcal{A}$-algebras.
\end{abstract}

Keywords Quasi-free resolutions · Koszul duality · Minimal models · Perturbation theory

Mathematics Subject Classification $18 \mathrm{D} 50 \cdot 16 \mathrm{~S} 15 \cdot 18 \mathrm{M} 70 \cdot 18 \mathrm{G} 55$

\section{Introduction}

Finding (possibly non-minimal) models for operads allows us understand the homotopy theory of their (co)algebras and, in particular, define their deformation complexes in the same spirit as is done with commutative, associative and Lie algebras, for example. In particular, finding a suitable model for an operad allows us to define its algebras up to homotopy, as it has been done, for example, for associative [37,38], commu-

Communicated by Jim Stasheff.

$凶$ Pedro Tamaroff

tamaroff@mis.mpg.de

1 Non-linear Algebra Group, Max-Planck-Institut für Mathematik in den Naturwissenschaften, Inselstraße 22, 04103 Leipzig, Germany 
tative, Lie [25], Gerstenhaber and Poisson [17], and BV algebras [14,16,40], among others.

It is well known that if $H$ is a bialgebra, the category of left $H$-modules admits an internal tensor product - the so called diagonal action of $H$-coming from its coproduct: if $V$ and $W$ are left $H$-modules, we define an action so that for each $h \in H$ and each $v \otimes w \in V \otimes W$, using Sweedler notation,

$$
h(v \otimes w)=h_{(1)} v \otimes h_{(2)} w .
$$

In this way, it makes sense to consider associative algebras in the category ${ }_{H}$ Mod of left $H$-modules. If $H$ is cocommutative, this category is symmetric monoidal, and it also makes sense to consider commutative associative algebras in the same monoidal category. Our first contribution is producing a functor

$$
H \longmapsto \text { Ass }_{H}
$$

that assigns to each weight graded bialgebra $H$ a weight graded operad $\mathrm{Ass}_{H}$ so that an associative algebra in ${ }_{H}$ Mod is the same as an Ass $_{H}$-algebra. The idea of producing functors from certain "amenable" categories to study operads and related structures, or even producing endofunctors on operads themselves, has already appeared several times in the literature, see [9-11] and [12, Chapter 4].

Unraveling the definitions, we see that the way the associative product $x_{1} x_{2}$ of an Ass $_{H}$-algebra and an operation $T_{h}$ coming from $h \in H$ behave with respect to each other is dictated by the coproduct of $H$ : using Sweedler notation, we require that

$$
T_{h}\left(x_{1} x_{2}\right)=T_{h_{(1)}}\left(x_{1}\right) T_{h_{(2)}}\left(x_{2}\right) .
$$

The first observation to make is that this relation is not quadratic, since the right hand side has three operators. As a result, the study of the operad Ass $_{H}$ falls outside the scope of the theory of Koszul duality, which is our go-to tool to study the homotopy theory of quadratic operads. To remedy this, we apply the methods of word operads of Dotsenko [10] and ideas coming from perturbation theory similar to those of Vallette and Merkulov [28, Theorem 40] to show how to obtain the minimal model of Ass $_{H}$ from an associated quadratic operad $q \mathrm{Ass}_{H}$ in case $H$ is Koszul. Moreover, we show how to construct a Gröbner basis of Ass $_{H}$ in case $H$ admits one, which shows in particular that $q \mathrm{Ass}_{H}$ admits a quadratic Gröbner basis in case $H$ admits one.

To build our intuition, one can think that this operad codifies the cases when the action of $H$ is trivial, effectively allowing us to split the study of $q \operatorname{Ass}_{H}$ into the study of $H$-modules and Ass-algebras: the minimal model of $q \mathrm{Ass}_{H}$ is generated by the desuspension of the ns sequence

$$
H^{i} \otimes \text { Ass }^{i}
$$

With this at hand, we apply techniques coming from pertubation theory coupled with an Ansatz coming from the theory of rewriting systems to describe the minimal model of $\mathrm{Ass}_{H}$, which is generated by the same ns sequence and incorporates some 
higher order terms in its differential. Our work will show that this perturbationalthough in general incorporating terms of arbitrarily high order-can be controlled completely through the Koszul model of $H$ and its coproduct.

Theorem Suppose that the underlying algebra of $H$ is Koszul. There exists a quasi-free resolution $\mathbb{M}\left(\operatorname{Ass}_{H}\right) \longrightarrow$ Ass $_{H}$ such that

- the underlying operad is of $\mathbb{M}\left(\mathrm{Ass}_{H}\right)$ is equal to underlying operad of the Koszul resolution $\mathbb{M}\left(\operatorname{gr~Ass}_{H}\right)$,

- the differential of $\mathbb{M}\left(\mathrm{Ass}_{H}\right)$ is obtained from the differential of $\mathbb{M}\left(\mathrm{grAss}_{H}\right)$ by a perturbation that lowers the weight induced by the external weight grading.

Moreover, this differential is homogeneous for the internal weight grading induced from $\mathrm{Ass}_{H}$, and the pertubation depends only on the Koszul differential and the iterated coproduct coming from $\mathrm{H}$.

The takeaway is that we can explicitly describe the differential of the minimal model of $\operatorname{Ass}_{H}$ provided we can do this for the Koszul model of $H$ and its coproduct. This problem, pertaining to the domain of algebras and coalgebras, is usually a simpler problem to tackle, so our result gives a useful bridge to solve from a much familiar problem a seemingly more complicated one. The theory of Koszul duality for usual associative algebras, on the other hand, has existed for almost five decades since its inception in [34], and now extensive literature and methods exist to deal with them and with many of their variants; see for example [13,20,24,26,33].

We also show that in case $H$ is Koszul and primitively generated, this operad does fall within a formalism to study pertubations of Koszul (pr)operads in [26] if the algebra underlying $H$ is Koszul, then Ass $_{H}$ is homotopy Koszul, and hence admits a minimal model of the same size of an associated Koszul (quadratic) operad $q \mathrm{Ass}_{H}$. It is important to point out that the main example we are interested in, the Steenrod algebra mod 2, does not fall within this more restrictive class of bialgebras, since it is not primitively generated. On the other hand, we can apply this last method to universal envelopes of Lie algebras, for example.

To illustrate our technique, we apply our methods to recover all the results of [6], where the author considers associative algebras with a tower of higher order derivations and describes its deformation complex. In this case, we show that the bialgebra controlling this operad is free as an algebra - which makes the determination of its Koszul dual and differential completely trivial - and give explicit formulas for its minimal model. Similar articles $[5,7,8]$ can be dealt with in the same way.

We also apply our formalism to the mod- 2 Steenrod algebra $\mathcal{A}$ : studying the category of associative algebras in the category of left $\mathcal{A}$-modules, usually called simply $\mathcal{A}$ algebras, and in particular, the so called unstable $\mathcal{A}$-algebras, is a classical problem in (unstable) homotopy theory. Our formalism assigns to each $\mathcal{A}$-algebra a deformation complex whose differential depends only on a very small part of the differential coming from the minimal model of $\operatorname{Ass}_{\mathcal{A}}$. It is important to note that if we instead want to consider the operad $\operatorname{Com}_{\mathcal{A}}$ controlling commutative algebras in $\mathcal{A}$-modules, we need to consider simplicial resolutions instead, since we are working with the commutative operad in positive characteristic $[4,15,31]$. We intend to pursue these ideas in the future. 
Notation and conventions. In what follows $\mathbb{k}$ is a field, and all unadorned $\otimes$ and hom are with respect to this base field. We assume the reader is familiar with the elements of operads as presented, for example, in [26, Chapters 5 and 6]. We are mainly interested in non-symmetric and, in particular, Ass is the non-symmetric operad governing associative algebras. We refer the reader to [26] for a useful and comprehensive reference on the basic theory of algebraic operads. With our mind in future applications, where we intend to replace Ass with a symmetric operad, we present our results in a way they easily generalize to this case.

\section{Some preliminaries on operads and algebras}

\subsection{Monomial orders and word operads}

Let us recall the essential facts from [10], that produces for us a functor $M \longmapsto \mathbb{W}_{M}$ from monoids to (symmetric) operads that, as explained there, can be used to produce useful orderings of shuffle tree monomials and, with them, Gröbner basis for operads.

Definition 2.1 Let $M$ be a monoid. We define the word operad $\mathbb{W}_{M}$ associated to $M$ so that, for each finite set $I$, we have $\mathbb{W}_{M}(I)=M^{I}$. For each map $f: I \longrightarrow \underline{n}$ we define

$$
\gamma_{f}: \mathbb{W}_{M}(\underline{n}) \times \mathbb{W}_{M}(f) \longrightarrow \mathbb{W}_{M}(I)
$$

so that for each $i \in I$, we have $\gamma_{f}\left(x ; y_{1}, \ldots, y_{n}\right)(i)=x(f(i)) y_{f(i)}(i)$.

Put differently, composing a tuple $x=\left(x_{1}, \ldots, x_{n}\right) \in \mathbb{W}_{M}(\underline{n})$ of elements with a tuple of elements $y=\left(y_{1}, \ldots, y_{m}\right) \in \mathbb{W}_{M}(\underline{m})$ at the $j$ th place, produces for us the element

$$
x \circ_{j} y=\left(x_{1}, \ldots, x_{j-1}, x_{j} y_{1}, \ldots, x_{j} y_{m}, x_{j+1}, \ldots, x_{n}\right) \in \mathbb{W}_{M}(\underline{m+n-1}) .
$$

The key feature of this functor is that it maps ordered monoids to ordered shuffle operads. To state the result, let us recall the following definitions.

Definition 2.2 An ordered monoid $M$ is a monoid equipped with a partial order for which the multiplication of $M$ is increasing in each of its arguments with respect to this order. Similarly, an ordered shuffle operad $\mathcal{P}$ is a shuffle operad so that for each $n \in \mathbb{N}$ the component $\mathcal{P}(n)$ comes equipped with a partial order in such a way that the composition map of $\mathcal{P}$ is increasing in each of its arguments with respect to these orders.

To illustrate, this condition means in particular that if we take two operations $\mu_{1} \prec$ $\mu_{2}$ of the same arity $t$ then for any tuple $\left(v_{1}, \ldots, v_{t}\right) \in \mathcal{P}\left(s_{1}\right) \times \cdots \times \mathcal{P}\left(s_{t}\right)$, we have that

$$
\gamma_{\mathcal{P}}\left(\mu_{1}, v_{1}, \ldots, v_{t}\right) \prec \gamma_{\mathcal{P}}\left(\mu_{2}, v_{1}, \ldots, v_{t}\right)
$$


for the corresponding partial order in $\mathcal{P}\left(s_{1}+\cdots+s_{t}\right)$. An analogous remark holds for the other arguments of $\gamma_{\mathcal{P}}$. In the case of a monoid $M$, we require that if $x, y, z \in M$ and $x \prec y$, then $x z \prec y z$ and $z x \prec z y$.

We can now state the following result, which we reproduce from Proposition 1.6 in [10]. It gives us a convenient gadget to produce ordered shuffle operads from ordered monoids. Naturally, producing the latter is a rather simpler task than producing the former.

Proposition 2.3 The functor $M \longmapsto \mathbb{W}_{M}$ restricts to a functor from ordered monoids to ordered shuffle operads if we endow $\mathbb{W}_{M}$ with the induced lexicographical order of tuples.

The ordered monoid that will be of interest to us is the monoid QM of "quantum monomials", as in [10, Theorem 2.1]. It is defined by

$$
\mathrm{QM}=\langle x, y, q \mid x y=q y x, q x=x q, q y=y q\rangle .
$$

Observe that each element in this monoid admits a unique expression in the form $x^{k} y^{l} q^{m}$ where $k, l, m \in \mathbb{N}$. Define an order so that $x^{k} y^{l} q^{m} \prec x^{k^{\prime}} y^{l^{\prime}} q^{m^{\prime}}$ if $k>k^{\prime}$ or $k=k^{\prime}$ and $l<l^{\prime}$, or $k=k^{\prime}$ and $l=l^{\prime}$ and $m<m^{\prime}$. Observe, in particular, that if $k>k^{\prime}$ then $x^{k} \prec x^{k^{\prime}}$.

Lemma 2.4 This order makes QM into an ordered monoid.

Proof See Lemma 2.2 in [10].

\subsection{Distributive laws and Koszul duality}

Let $\mathcal{P}=\mathcal{F}(V, R)$ be a quadratic operad, and let us recall from [26, Definition 7.4.4, Theorem 7.4.6] the following definition (or theorem, depending on the preference of the reader):

Definition 2.5 The operad $\mathcal{P}$ is Koszul if and only if the homology of its bar construction $B(\mathcal{P})$ is concentrated in syzygy degree zero.

We remind the reader that $B(\mathcal{P})$ is the free cooperad on the suspension $s \overline{\mathcal{P}}$ of the kernel $\overline{\mathcal{P}}$ of the augmentation of $\mathcal{P}$, and that the syzygy degree of an element $x \in B(\mathcal{P})$ is the difference $n_{1}-n_{2}$ where $n_{1}$ is the homological degree of $x$ and $n_{2}$ is the total weight of elements appearing in its internal vertices.

We now recollect the standard facts about distributive laws [1,27], these are useful algebraic gadgets that allow us to decompose a given operad $\mathcal{P}$ into two constituent suboperads $\mathcal{P}^{\prime}$ and $\mathcal{P}^{\prime \prime}$. Once this is done, information about $\mathcal{P}^{\prime}$ and $\mathcal{P}^{\prime \prime}$ can be translated into information about $\mathcal{P}$.

Definition 2.6 Let $\mathcal{P}^{\prime}$ and $\mathcal{P}^{\prime \prime}$ be ns operads. A distributive law is a map

$$
\lambda: \mathcal{P}^{\prime \prime} \circ \mathcal{P}^{\prime} \longrightarrow \mathcal{P}^{\prime} \circ \mathcal{P}^{\prime \prime}
$$


for which the following identities hold:

$$
\lambda\left(1 \circ \gamma_{\mathcal{P}^{\prime}}\right)=\left(\gamma_{\mathcal{P}^{\prime \prime}} \circ 1\right)(1 \circ \lambda)(\lambda \circ 1), \quad \lambda\left(\gamma_{\mathcal{P}^{\prime \prime}} \circ 1\right)=\left(1 \circ \gamma_{\mathcal{P}^{\prime}}\right)(\lambda \circ 1)(1 \circ \lambda) .
$$

We also require that $\lambda$ respects the units: $\lambda\left(1 \circ \eta_{\mathcal{P}^{\prime}}\right)=\eta_{\mathcal{P}^{\prime \prime}} \circ 1$ and $\lambda\left(\eta_{\mathcal{P}^{\prime \prime}} \circ 1\right)=1 \circ \eta_{\mathcal{P}^{\prime}}$. The following is proven in [26, Proposition 8.6.2].

Proposition 2.7 If $\lambda$ is a distributive law between $\mathcal{P}^{\prime}$ and $\mathcal{P}^{\prime \prime}$ then the sequence $\mathcal{P}^{\prime} \circ \mathcal{P}^{\prime \prime}$ admits a composition making it an operad given by

$$
\gamma_{\lambda}=\left(\gamma_{\mathcal{P}^{\prime \prime}} \circ \gamma_{\mathcal{P}^{\prime}}\right)(1 \circ \lambda \circ 1)
$$

One of the useful features of distributive laws is they behave well with Koszul duality when they are obtained from a distributive law for the respective quadratic data. To define these, let us take quadratic presentations $\mathcal{P}^{\prime}=\mathcal{F}\left(V^{\prime}\right) /\left(R^{\prime}\right)$ and $\mathcal{P}^{\prime \prime}=$ $\mathcal{F}\left(V^{\prime \prime}\right) /\left(R^{\prime \prime}\right)$. A rewriting rule between $V$ and $W$ is a map

$$
\lambda: V^{\prime \prime} \circ_{(1)} V^{\prime} \longrightarrow V^{\prime} \circ_{(1)} V^{\prime \prime}
$$

Consider the graph $R_{\lambda}=\left\{t-\lambda(t): t \in V^{\prime \prime} \circ_{(1)} V^{\prime}\right\} \subseteq \mathcal{F}\left(V^{\prime} \oplus V^{\prime \prime}\right)^{(2)}$. We define the quadratic operad

$$
\mathcal{P}=\mathcal{P}^{\prime} \vee_{\lambda} \mathcal{P}^{\prime \prime}=\mathcal{F}\left(V \oplus V^{\prime}\right) /\left(R^{\prime}, R^{\prime \prime}, R_{\lambda}\right) .
$$

One can check that we have maps of sequences

$$
\pi: \mathcal{P}^{\prime} \circ \mathcal{P}^{\prime \prime} \longrightarrow \mathcal{P}, \pi^{\prime}: \mathcal{P}^{\prime \prime} \circ \mathcal{P}^{\prime} \longrightarrow \mathcal{P} \text {. }
$$

Proposition 2.8 If the map $\pi$ is an isomorphism of sequences, then the composition $\lambda=\pi^{-1} \pi^{\prime}: \mathcal{P}^{\prime \prime} \circ \mathcal{P}^{\prime} \longrightarrow \mathcal{P}^{\prime} \circ \mathcal{P}^{\prime \prime}$ defines a distributive law between $\mathcal{P}^{\prime}$ and $\mathcal{P}^{\prime \prime}$, and the map $\pi$ determines an isomorphism of operads.

The shinning example of an operad obtained from a distributive law is the following. Let us consider the commutative operad Com and the Lie operad Lie where $V^{\prime}=\langle\mu\rangle$ is generated by a symmetric associative operation $x_{1} x_{2}$ and $V^{\prime \prime}=\langle\beta\rangle$ is generated by an antisymmetric Lie bracket $\left[x_{1}, x_{2}\right]$. Consider the rewriting rule defined by

$$
\left[x_{1} x_{2}, x_{3}\right] \in V^{\prime \prime} \circ_{(1)} V^{\prime} \longmapsto x_{1}\left[x_{2}, x_{3}\right]+x_{2}\left[x_{1}, x_{3}\right] \in V^{\prime} \circ_{(1)} V^{\prime \prime} \text {. }
$$

The Poisson operad is exactly given by Com $\vee_{\lambda}$ Lie, and one can show, by a cardinality argument, that the map $\pi:$ Com $\circ$ Lie $\longrightarrow$ Pois is indeed an isomorphism. The following result then implies that the Poisson operad is Koszul, see [26, Proposition 8.6.9]:

Lemma 2.9 If $\mathcal{P}^{\prime}$ and $\mathcal{P}^{\prime \prime}$ are Koszul operads any operad $\mathcal{P}$ obtained from them through a distributive law between their quadratic data is also Koszul, and conversely. 
We also recall that Gröbner basis criterion to check for Koszulness.

Proposition 2.10 Any operad that admits a quadratic Gröbner basis is Koszul.

\subsection{Perturbation theory}

Let us now consider a weight graded operad $\mathcal{P}=\mathcal{F}(W) /(S)$ where the space of relations is contained in $\mathcal{F}(W)^{\geq 2}$. Let us also write $S_{2}=\pi_{2}(S)$ where $\pi_{2}: \mathcal{F}(W) \longrightarrow$ $\mathcal{F}(W)^{(2)}$ is the projection. With this at hand, we consider a class of presentations that are obtained, in a precise sense, by taking a Koszul operad and adding "higher order terms" to the relations:

Definition 2.11 We say that a presentation of a weight graded operad $\mathcal{P}=\mathcal{F}(W) /(S)$ is homotopy Koszul if the following three conditions hold:

(1) The quadratic operad $q \mathcal{P}=\mathcal{F}(W) /\left(S_{2}\right)$ is Koszul.

(2) The ns sequences $q \mathcal{P}$ and $\mathcal{P}$ are isomorphic.

(3) There is an extra grading on $\mathcal{P}$ by finite dimensional symmetric sequences.

Let us write $\mathbb{M}(\mathcal{P})$ for a choice of minimal quasi-free resolution $\mathbb{M}(\mathcal{P}) \longrightarrow \mathcal{P}$ in the category of weight graded operads.

Theorem 2.12 If a $\mathcal{P}$ admits a homotopy Koszul presentation, then it also admits a weight graded minimal model $\mathbb{M}(\mathcal{P})$ such that:

- the underlying free operad of $\mathbb{M}(\mathcal{P})$ is equal to that of $\mathbb{M}(q \mathcal{P})$ and,

- the differential is obtained from the differential of $\mathbb{M}(q \mathcal{P})$ that lowers the filtration degree induced by the extra weight grading.

Proof See Theorem 40 in [28].

To illustrate the idea of these "perturbative" methods at the level of associative algebras, let us consider the algebra GP $=T V /(R)$ of cohomology operations of Singer [36, Section 5]. It is defined over $\mathbb{F}_{2}$ by a countable family of generators $t, s_{0}, s_{1}, s_{2}, \ldots$ of respective degrees $1,1,2,4, \ldots$, along with a family of homogeneous relations $R$ whose leading quadratic terms $R_{2}$ are the following

$$
s_{i}^{2}, \quad\left[s_{0}, t\right]=t^{2}, \quad\left[s_{i}, s_{j}\right]=0, \quad\left[s_{i+1}, t\right]=0 \quad \text { for } j>i \geq 0 .
$$

By further discarding the term $t^{2}$-for example, by considering the lexicographical order where $t<s_{0}<s_{1}<\cdots$-we see that the quadratic algebra $q \mathrm{GP}=T(V) /\left(R_{2}\right)$ is Koszul. Since both GP and $q$ GP admit linear bases given by "admissible" monomials [36, Lemma 5.3]

$$
t^{k} s_{i_{1}} \cdots s_{i_{r}}, \quad \text { for } k \geq 0 \text { and } 0 \leq i_{1}<\cdots<i_{r}
$$

we see that Singer's algebra GP is homotopy Koszul. The main result in that section is a determination of the Quillen homology of GP [36, Proposition 5.6]. 
Recall we write $\mathbb{M}(\mathcal{P})$ for a choice of minimal quasi-free resolution $\mathbb{M}(\mathcal{P}) \longrightarrow \mathcal{P}$ in the category of (weight) graded operads. The following theorem shows that if $\mathcal{P}$ is an operad for which we know $\mathbb{M}(\mathcal{P})$ and if $\mathcal{Q}$ is another operad with the same underlying symmetric sequence as $\mathcal{P}$, then we can produce $\mathbb{M}(\mathcal{Q})$ from $\mathbb{M}(\mathcal{P})$. Our main interest lies in the case when $\mathcal{P}$ is Koszul, and $\mathcal{Q}$ can be filtered in such a way that the graded operad associated to $\mathcal{Q}$ is equal to $\mathcal{P}$.

Theorem 2.13 Suppose that $\mathcal{P}$ and $\mathcal{Q}$ are isomorphic as symmetric sequences. Then there exists a quasi-free resolution $\mathbb{M}(\mathcal{Q}) \longrightarrow Q$ such that:

- the underlying free operad of $\mathbb{M}(2)$ is equal to that of $\mathbb{M}(\mathcal{P})$ and,

- the differential is obtained from the differential of $\mathbb{M}(\mathcal{P})$ by a perturbation that lowers the filtration degree induced by the weight grading.

Proof See the Appendix of [22].

\section{The functor from bialgebras to operads}

Let us consider a weight graded locally finite bialgebra $H$ with conilpotent comultiplication $\Delta$. We define a map $\lambda: H \circ$ Ass $\longrightarrow$ Ass $\circ H$ so that for each $h \in H$ and each $n \in \mathbb{N}$ we have that

$$
\lambda\left(h \otimes \mu_{n}\right)=\mu_{n} \circ\left(h_{(1)}, \ldots, h_{(n)}\right)
$$

where we use Sweedler notation on the right for the iterated coproduct $\Delta^{(n)}$ of $H$. In what follows, it will be useful to note that $H \circ$ Ass $=H \otimes$ Ass since $H$ is concentrated in arity one.

Lemma 3.1 The map $\lambda$ induces distributive law between the associative operad Ass and the associative algebra underlying $H$.

Proof We have to check that the composition

$$
H \otimes H \otimes \text { Ass } \stackrel{1 \otimes \lambda}{\longrightarrow} H \otimes(\text { Ass } \circ H) \stackrel{\lambda \otimes 1}{\longrightarrow} \text { Ass } \circ(H \otimes H) \stackrel{1 \circ \mu_{H}}{\longrightarrow} \text { Ass } \circ H
$$

is equal to the composition $H \otimes H \otimes$ Ass $\stackrel{\mu_{H} \otimes 1}{\longrightarrow} H \otimes$ Ass $\stackrel{\lambda}{\longrightarrow}$ Ass $\circ H$. This is a direct verification: the first map assigns $h^{\prime} \otimes h^{\prime \prime} \otimes \mu_{n}$ to $\mu_{n} \circ\left(h_{(1)}^{\prime} h_{(1)}^{\prime \prime}, \ldots, h_{(n)}^{\prime} h_{(n)}^{\prime \prime}\right)$ while the second one assigns it to $\mu_{n} \circ\left(\left(h^{\prime} h^{\prime \prime}\right)_{(1)}, \ldots,\left(h^{\prime} h^{\prime \prime}\right)_{(n)}\right)$, and this two terms are equal since $\Delta$ is a map of associative algebras. We also have to check that the composition

$$
(H \otimes \text { Ass }) \circ \text { Ass } \stackrel{\lambda \circ 1}{\longrightarrow} \text { Ass } \circ(H \otimes \text { Ass }) \stackrel{1 \circ \lambda}{\longrightarrow}(\text { Ass } \circ A s s) \circ H \stackrel{\gamma_{\text {Ass }} \circ 1}{\longrightarrow} \text { Ass } \circ H
$$

is equal to the composition $H \otimes$ (Ass $\circ$ Ass) $\stackrel{1 \otimes \gamma_{\text {Ass }}}{\longrightarrow} H \otimes$ Ass $\stackrel{\lambda}{\longrightarrow}$ Ass $\circ H$. In this case, the first map assigns the element $h \otimes \mu_{n} \circ \mu_{m}$ to $\mu_{m+n} \circ \Delta^{(n)} \Delta^{(m)}(h)$ while the second map assigns it to the element $\mu_{m+n} \circ \Delta^{(n+m)}(h)$. In view of the coassociativity of $\Delta$, these two elements coincide. 
From this we obtain an operad which we write $\operatorname{Ass}_{H}$, with underlying symmetric sequence $\left(\operatorname{Ass}_{H}\right)^{\#}=$ Asso $H$. We will call an Ass ${ }_{H}$-algebra an associative $H$-algebra. As mentioned in the introduction, it is easy to see that:

Proposition 3.2 The category of Ass $_{H}$-algebras and the category of associative algebras in left $\mathrm{H}$-modules are isomorphic.

Our objective is to obtain the minimal model of $\operatorname{Ass}_{H}$. It is important to observe that $\operatorname{Ass}_{H}$ is defined by quadratic-cubic relations unless the comultiplication of $H$ is trivial; as such, it falls outside the scope of the theory of Koszul duality. To remedy this, we consider two approaches:

- the formalism of word operads developed by Dotsenko in [10] and some elementary perturbation theory, which we recalled in Sects. 2.1 and 2.3.

- the notion of homotopy Koszul (pr)operads due to Merkulov and Vallette in [28, Section 5.4, Theorem 40].

Running conventions In what follows, we will fix a presentation $H=T(V) /(R)$ of $H$. In this way, we obtain a presentation of $\operatorname{Ass}_{H}$ of the form

$$
\operatorname{Ass}_{H}=\mathcal{F}(\mathbb{k} \mu \oplus V) /\left(R_{\mu}, R_{\lambda}, R\right)
$$

where $R_{\mu}$ is the associativity relation of $\mu$ and $R_{\lambda}$ is the set of relations between $v \in V$ and $\mu$ induced by the distributive law $\lambda: H \otimes$ Ass $\longrightarrow$ Ass $\circ H$. We will mainly be concerned in the situation when $R$ is quadratic or linear-quadratic. The operad $\operatorname{Ass}_{H}$ inherits an internal weight grading (compatible with the operad structure) from that of $H$, and admits a filtration degree (for which its relations are not homogeneous) in which we put $H$ in weight one.

\subsection{Main results}

Recall that $\mathbb{W}_{\mathrm{QM}}$ is the word operad associated to the monoid of quantum monomials, and that $\mathcal{F}(\mathbb{k} \mu \oplus V)$ is the free operad generated by a binary operation $\mu$ and the space of generators $V$ of the underlying algebra of $H$.

Define a map $f: \mathcal{F}(\mathbb{k} \mu \oplus V) \longrightarrow \mathbb{W}_{\mathrm{QM}}$ such that for each $v \in V$,

$$
f(v)=(y), \quad f(\mu)=(x, x) .
$$

Since the operad $\mathbb{W}_{\mathrm{QM}}^{s h}$ is ordered, we obtain an order in $\mathcal{F}^{s h}(\mathbb{k} \mu \oplus V)$ by comparing images in $\mathbb{W}_{\mathrm{QM}}^{s h}$. We extend it to a proper monomial order by superposition with the path-lexicographic order. Let us call the resulting monomial order the quantum order.

Lemma 3.3 The leading terms of the defining relations of the operad

$$
\operatorname{Ass}_{H}=\mathcal{F}(\mathbb{k} \mu \oplus V) /\left(R_{\mu}, R_{\lambda}, R\right)
$$

for the quantum order are: 
- the tree monomials $T_{v}\left(x_{1} x_{2}\right)$ for $v \in V$, the generators of $H$,

- the tree monomial $x_{1}\left(x_{2} x_{3}\right)$ of the associative operad Ass,

- the leading monomials of the defining relations $R$ of $T V$.

Proof The relators contained in $R_{\lambda}$ are of the form

$$
T_{v}\left(x_{1} x_{2}\right)-T_{v_{(1)}}\left(x_{1}\right) T_{v_{(2)}}\left(x_{2}\right)
$$

for $v \in V$. The first summand, which is just $v \circ_{1} \mu$, maps to $y \circ_{1}(x, x)=(y x, y x)$ while the other summands map to $\gamma((x, x) ; y, y)=(x y, x y), \gamma((x, x): 1, y)=(x, x y)$ or $\gamma((x, x): 1, y)=(x y, x)$. The way we have constructed QM implies that $x y q=y x$ is larger than $x$ and $x y$. On the other hand, the terms $\mu \circ_{1} \mu$ and $\mu \circ_{2} \mu$ map, respectively, to $(x, x) \circ_{1}(x, x)=(x x, x x, x)$ and $(x, x) \circ_{2}(x, x)=(x, x x, x x)$, and the second is larger than the first since $x x<x$. Finally, it is clear that the leading monomials in the relations $R$ defining $H$ are unaffected.

Corollary 3.4 If the defining relations $R$ form a Gröbner basis for $H$, then the defining relations $R \cup R_{\lambda} \cup R_{\mu}$ form a Gröbner basis for Ass $_{H}$.

Proof The normal forms for the leading terms of the previous lemma are:

- the normal forms of $H$,

- the left combs in $\mathcal{F}(\mu)$,

- the the left combs in $\mathcal{F}(\mu)$ with normal forms of $H$ on their leaves.

This already accounts for every single element of $\left(\text { Ass }_{H}\right)^{\#}=$ Ass $\circ H$, so we have a Gröbner basis. Indeed, if we had a non-zero term for an $S$-polynomial, it would create for us a linear dependence relation between normal forms, which cannot be.

Let us now assume that the relations of $R$ are quadratic, and write $q \mathrm{Ass}_{H}$ for the operad $q \operatorname{Ass}_{H}=\mathcal{F}(\mathbb{k} \mu \oplus V) /\left(R_{\mu}, R_{\lambda}^{\prime}, R\right)$ where we have just kept the leading tree monomials in $R_{\lambda}$ as described in Lemma 3.3. Observe that it is a quadratic operad which is obtained from Ass and $H$ by the trivial distributive law. The following is immediate from Lema 2.9 and from the analysis done in Corollary 3.4.

Corollary 3.5 If H is Koszul, then so is the associated quadratic operad qAss ${ }_{H}$. Moreover, if the defining relations $R$ form a quadratic Gröbner basis, the defining relations $R \cup R_{\lambda}^{\prime} \cup R_{\mu}$ form a quadratic Gröbner basis for $q$ Ass $_{H}$.

It is clear that the underlying symmetric sequence of $\operatorname{grAss}_{H}$ and $\mathrm{Ass}_{H}$ are isomorphic: they are both isomorphic to Ass $\circ H$. In particular, if $H$ is Koszul, then the Koszul dual cooperad to gr $\operatorname{Ass}_{H}$ has underlying sequence given by

$$
\left(q \operatorname{Ass}_{H}\right)^{i, \#}=H^{i} \otimes \text { Ass }^{i} .
$$

From Theorem 2.13, we deduce our main theorem.

Theorem 3.6 Suppose that the underlying algebra of $H$ is Koszul. There exists a quasifree resolution $\mathbb{M}\left(\mathrm{Ass}_{H}\right) \longrightarrow \mathrm{Ass}_{H}$ such that 
- the underlying operad is of $\mathbb{M}\left(\mathrm{Ass}_{H}\right)$ is equal to underlying operad of the Koszul resolution $\mathbb{M}\left(\operatorname{gr~Ass}_{H}\right)$,

- the differential of $\mathbb{M}\left(\mathrm{Ass}_{H}\right)$ is obtained from the differential of $\mathbb{M}\left(g r \mathrm{Ass}_{H}\right)$ by a perturbation that lowers the filtration degree induced by the external weight grading.

Moreover, this differential is homogeneous for the internal weight grading induced from Ass $_{H}$, and the pertubation depends only on the Koszul differential and the iterated coproduct coming from $H$, and increases the filtration degree.

\subsection{The primitively generated case}

In case $H$ is primitively (finitely) generated - for example, in case we are working over a field of characteristic zero and $H$ is connected, cocommutative and locally finite [30, Theorem 5.18] — we have the following result, which provides us with a shortcut to obtain the theorem above, that by-passes the (perhaps technical) constructions involving word operads.

Theorem 3.7 If the underlying algebra of $H$ is Koszul and primitively finitely generated, then the operad Ass $_{H}$ is homotopy Koszul in the sense of Merkulov-Vallette.

Proof Let us write Ass $_{H}=\mathcal{F}(W) /(S)$. To show it is homotopy Koszul, we need to verify the three conditions in Definition 2.11.

The quadratic operad $q \mathrm{Ass}_{H}$ is easily described: it consists of an associative operation $\mu$ and one operation $T_{h}$ for each $h \in \operatorname{Prim}(H)$ subject to the condition that each of these is a derivation for $\mu$ :

$$
T_{h}\left(x_{1} x_{2}\right)=T_{h}\left(x_{1}\right) x_{2}+x_{1} T_{h}\left(x_{2}\right)
$$

This operad is then obtained from a distributive law between the Koszul algebra $H$ and the Koszul operad Ass, and is thus Koszul by Lemma 2.9, with underlying symmetric sequence isomorphic to Ass $H$. By construction, the ns sequence underlying $\operatorname{Ass}_{H}$ is isomorphic to Ass $\circ H$. The third condition holds since we assume $H$ is locally finite dimensional. This gives Ass $_{H}$ an internal weight grading which is also locally finite dimensional, since the distributive law preserves the weight grading of $H$.

Theorem 3.8 Suppose that the underlying algebra of $H$ is Koszul and primitively generated. There exists a quasi-free resolution $\mathbb{M}\left(\operatorname{Ass}_{H}\right) \longrightarrow \operatorname{Ass}_{H}$ such that

- the underlying operad is of $\mathbb{M}\left(\mathrm{Ass}_{H}\right)$ is equal to underlying operad of the Koszul resolution $\mathbb{M}\left(q \operatorname{Ass}_{H}\right)$,

- the differential of $\mathbb{M}\left(\mathrm{Ass}_{H}\right)$ is obtained from the differential of $\mathbb{M}\left(q \mathrm{Ass}_{H}\right)$ by a perturbation that lowers the filtration degree induced by the additional weight grading.

Moreover, this differential is homogeneous for the internal weight grading induced from $\mathrm{Ass}_{H}$, and the pertubation depends only on the Koszul differential and the iterated coproduct coming from $H$. 
As we mentioned in the introduction, the conclusion is that the minimal model of Ass $_{H}$ can be described entirely by familiar objects: the Koszul model of $q$ Ass $_{H}$, that of the algebra $H$, and the comultiplication of $H$.

Proof We need only address the shape of the differential of the minimal model of $\operatorname{Ass}_{H}$. Following [28], we recall one obtains the differential $\delta$ as a perturbation $\delta^{\prime}+\delta^{\prime \prime}$ of $\delta^{\prime}$, the Koszul differential of $q$ Ass $_{H}$. The term $\delta^{\prime \prime}$ appears by iterating the rewriting rule we introduced in Lemma 3.1. More precisely, we can consider the external weight grading of Ass $_{H}$ obtained by putting $H$ in weight 1 , so that this rewriting rule increases the weight exactly by one. The conilpotence condition on $\Delta$ means this process will eventually stop, and then the perturbation $\delta^{\prime}$ will consist of a locally finite sum, where the term $\delta_{i}$ will depend only on the iteration $\Delta^{(i)}$ of $\Delta$ and the Koszul differential of $H$, as we claimed.

\subsection{The deformation complex}

Let $A$ be an associative algebra in $H$-modules, and let us recall that the (homotopy type of) deformation complex of $A$ as an (usual) associative algebra can be computed as

$$
\operatorname{Def}(\mathrm{id}: A \longrightarrow A)=\operatorname{Der}(\Omega B A, A)=\operatorname{hom}(\overline{B A}, A)[-1]
$$

where $B A=T^{c}(s \bar{A})$ is the bar construction on $A$, and the differential is given by the multiplication of $A$ plus a term $[\pi,-]$ obtain from the canonical twisting cochain $\pi: B A \longrightarrow A$. More generally, if $B \longrightarrow A$ is a cofibrant model of $A$ in associative algebras, one can compute the deformation complex above as the dg Lie algebra of derivations

$$
\operatorname{Def}(\mathrm{id}: A \longrightarrow A)=\operatorname{Der}(B) \text {. }
$$

The homology of this complex is almost equal to the Hochschild cohomology of $A$ : we have that

$$
H^{n} \operatorname{Def}(\mathrm{id}: A \longrightarrow A)= \begin{cases}\operatorname{HH}^{n+1}(A) & \text { if } n \geq 1 \\ \operatorname{Der}(A) & \text { if } n=0\end{cases}
$$

More generally, we can consider a map of associative algebras $f: A^{\prime} \longrightarrow A$, and then

$$
H^{n} \operatorname{Def}\left(f: A^{\prime} \longrightarrow A\right)= \begin{cases}\operatorname{HH}^{n+1}\left(A^{\prime}, A\right) & \text { if } n \geq 1 \\ \operatorname{Der}\left(A^{\prime}, A\right) & \text { if } n=0\end{cases}
$$

Since it will do us no harm, let us work instead with the unital version of the deformation complex, which we write $C^{*}(A)$. It has underlying complex hom $(B A, A)$ and the usual Hochschild differential, and we write $\mathrm{HH}^{*}(A)$ for its cohomology groups. 
Similarly, we write $\mathrm{HH}^{*}(H \mid A)$ for the cohomology groups of $A$ when viewed as an $H$-algebra. Let us now show how to relate these two objects.

Lemma 3.9 Let $A$ be an $H$-algebra. Then the bar construction B A is a dga coalgebra in left $\mathrm{H}$-modules.

Proof The action of $H$ on $B A$ is obtained through the diagonal action on the individual summands $A^{\otimes n}$. The fact that this action commutes with the differential and the coalgebra structure of $B A$ is just a restatement of Lemma 3.1.

From the lemma it follows that $C^{*}(A)$ is left dg $H$-module. If we focus our attention back on the generators $H^{i} \otimes$ Ass $^{i}$ of the model of $\operatorname{Ass}_{H}$, we observe that the unital deformation complex of an $H$-algebra $A$ has the form

$$
C_{H}^{*}(A)=\operatorname{hom}\left(H^{i} \otimes B A, A\right)=\operatorname{hom}\left(H^{i}, C^{*}(A)\right) .
$$

Ignoring higher order terms in this differential, we obtain the Koszul complex computing the functor $\operatorname{Ext}_{H}^{*}(\mathbb{k},-)$. This observation leads to the following result:

Theorem 3.10 Let A be an H-algebra. Then the Hochschild cohomology groups $H H^{*}(A)$ are left $H$-modules, and there is a first quadrant spectral sequence

$$
\operatorname{Ext}_{H}^{p}\left(\mathbb{k}, H H^{q}(A)\right) \stackrel{p}{\Longrightarrow} H H^{p+q}(H \mid A)
$$

converging to the cohomology of $A$ as an $H$-algebra.

Proof We consider the filtration degree on $\mathbb{M}\left(\mathrm{Ass}_{H}\right)$ from Theorem 3.6, which induces a filtration on the deformation complex $C_{H}^{*}(A)$. In the 0th page, the differential is induced by that of $C^{*}(A)$, since the remaining terms of the differential contain elements of $H$ and hence increase the filtration degree. It follows that

$$
E_{1}=\operatorname{hom}\left(H^{!}, \mathrm{HH}^{*}(A)\right)
$$

with an induced differential. One can check that this is given by the differential of the Koszul complex for $\mathrm{HH}^{*}(A)$, since the remaining terms increase the filtration degree at least by two. Hence, we have that

$$
E_{2}=\operatorname{Ext}_{H}^{*}\left(\mathbb{k}, \mathrm{HH}^{*}(A)\right) \text {. }
$$

Since this spectral sequence is of first quadrant, convergence is guaranteed.

\subsection{Operads arising from coalgebras}

Let us now consider the simpler case that $C$ is a weight graded coalgebra and form the bialgebra $H=T(C)$ : the product is free, and the coproduct is the unique coproduct that restrict to that $C$ and makes $H$ into a bialgebra; in the literature, this bialgebra is known as the Hopf algebra of noncommutative symmetric functions, see $[19,32]$. 
Then $H^{i}=\mathbb{k} \oplus C$ is the trivial coalgebra, and its Koszul differential is trivial, so we can in fact give a very explicit description of the differential of Ass ${ }_{H}$. Let us first record the formula for the Koszul part $\delta_{1}$ of the differential.

Lemma 3.11 The weight zero part of the differential in the minimal model $\mathbb{M}\left(\right.$ Ass $\left._{H}\right)$ is such for each $n \in \mathbb{N}$,

$$
\begin{aligned}
\delta_{1}\left(1 \otimes \mu_{n}\right)= & \sum_{s+t=n+1}(-1)^{(i+1) t+n}\left(1 \otimes \mu_{s}\right) \circ_{i}\left(1 \otimes \mu_{t}\right) \\
\delta_{1}\left(c \otimes \mu_{n}\right)= & -\sum_{s+t=n+1}(-1)^{(i+1) t+n} \\
& \times\left(\left(c \otimes \mu_{s}\right) \circ_{i}\left(1 \otimes \mu_{t}\right)+(-1)^{t}\left(1 \otimes \mu_{s}\right) \circ_{i}\left(c \otimes \mu_{t}\right)\right) .
\end{aligned}
$$

Let us now address the higher weight part if the differential. To do this, let us fix an element $c \otimes \mu_{n}$ and some $j \geq 1$, and introduce some useful definitions and notation. We say the tuple $\vec{k}=\left(k_{0}, \ldots, k_{j}\right)$ is admissible if $k_{0} \geq j$ and

$$
|\vec{k}|:=k_{0}+\cdots+k_{j}=n+j
$$

Let us also define, for any tuple $\vec{m}=\left(m_{1}, \ldots, m_{j}\right)$ of positive integers and operations $v_{0}, v_{1} \ldots, v_{j}$ with $v$ of arity $|v|$ at least $|\vec{m}|$

$$
v_{0} \circ_{\vec{m}}\left(v_{1}, \ldots, v_{j}\right)=v_{0}\left(1^{m_{1}-1}, v_{1}, \ldots, 1^{m_{j}-1}, v_{j}, 1^{|v|-|\vec{m}|}\right)
$$

For such an admissible tuple $\vec{k}$, we define the operation

$$
v\left(\mu_{n}, c, \vec{k}\right)=\sum_{|\vec{m}|=k_{0}-j}(-1)^{\varepsilon(\vec{m}, \vec{k})} \mu_{k_{0}} \circ \vec{m}\left(c_{(1)} \otimes \mu_{k_{1}}, \ldots, c_{(j)} \otimes \mu_{k_{j}}\right) .
$$

Put differently, we consider all possible ways of placing the operations

$$
c_{(1)} \otimes \mu_{k_{1}}, \ldots, c_{(j)} \otimes \mu_{k_{j}}
$$

on the leaves of $\mu_{k_{0}}$ in this order. Note that the first admissibility condition means there will always be room to do this (although there may be no free slot at the end) and the second condition implies the arity of the resulting operation is the same than that of $c \otimes \mu_{n}$. With this at hand, we have the following result:

Theorem 3.12 For each $c \in C$ and each $n, j \geq 2$, we have that

$$
\delta_{j}\left(c \otimes \mu_{n}\right)=\sum_{\vec{k} \text { adm }}(-1)^{\delta(n, \vec{k})} v\left(c, \mu_{n}, \vec{k}\right)
$$

where the sum runs through admissible tuples of length $j+1$.

Remark that the signs $\varepsilon(\vec{m}, \vec{k})$ and $\delta(n, \vec{k})$ can be worked out explicitly. 
Proof Let us observe that $\delta^{2}=0$, which shows that this is the minimal model of $\operatorname{Ass}_{H}$. To do this, we will show that the elements in $\delta^{2}$ appear in pairs that cancel. Although we didn't make the signs $\delta$ and $\varepsilon$ explicit, this in fact shows that these signs can be chosen in a coherent way so that $\delta^{2}=0$.

Let us then fix some element $c \otimes \mu_{n}$ and consider the terms appearing in $\delta^{2}\left(c \otimes \mu_{n}\right)$ by picking some $s, t \in \mathbb{N}$ and looking at the terms appearing in

$$
\delta_{1} \delta_{s+t-1}, \quad \delta_{s+t-1} \delta_{1}, \quad \text { and } \delta_{s} \delta_{t} .
$$

When we apply $\delta_{1} \delta_{s+t-1}$, we will obtain a sum with terms of the following six shapes, where we use a dark dot to denote an element of the form $c^{\prime} \otimes \mu_{k}$ with $c^{\prime} \in C$ and a light dot to denote an element of the form $1 \otimes \mu_{j}$, and we use a dotted edge to mark where we have applied a differential $\delta_{0}$ in the last step. The figures arise as follows: we applied $\delta_{0}$ on a term of Ass and more than one dark dot was grafted on the leaves of the resulting operation, exactly one dot was placed in the resulting operation, we applied $\delta_{0}$ to a dark dot above a light one, we applied $\delta_{0}$ to a light dot and every dark dot was grafted on the leaves, we applied $\delta_{0}$ to a dark dot above a light one and, finally, we applied $\delta_{0}$ to a light dot and no dark dot was grafted on its leaves:
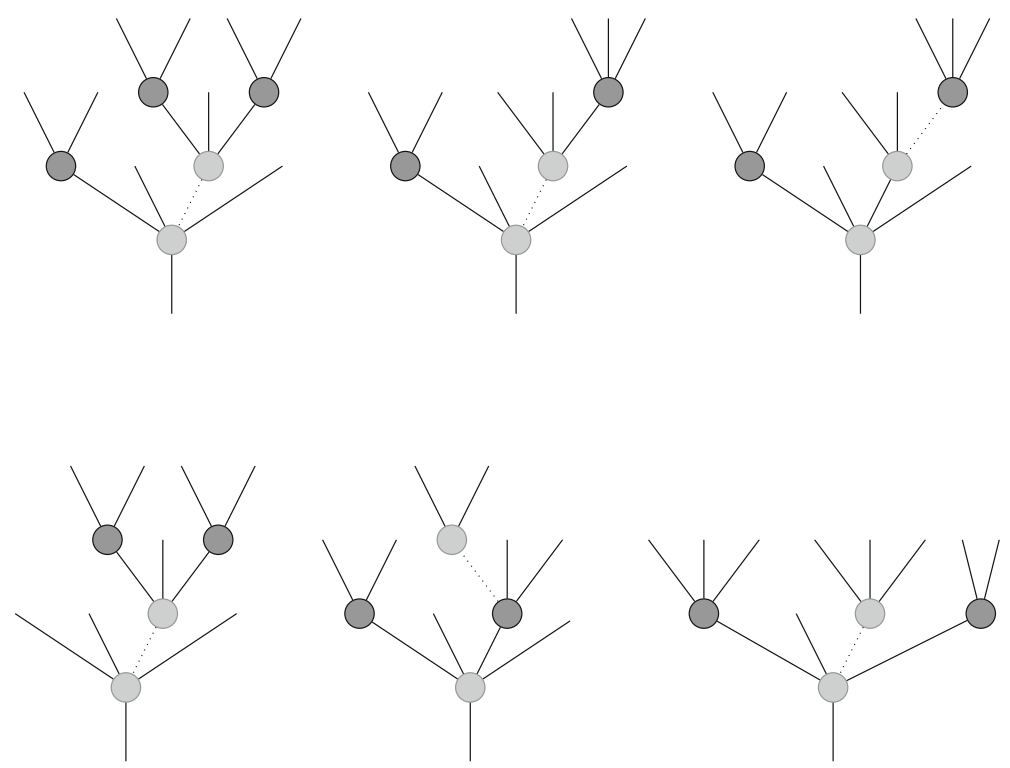

Applying $\delta_{s+t-1} \delta_{1}$ will produce for us a sum with terms of the following three shapes depending on whether: we apply $\delta_{s+t-1}$ to a lone dark dot above a light one, we apply $\delta_{s+t-1}$ to a lone dark dot below a light one and this light dot falls on a dark dot, and finally if it falls on an identity: 

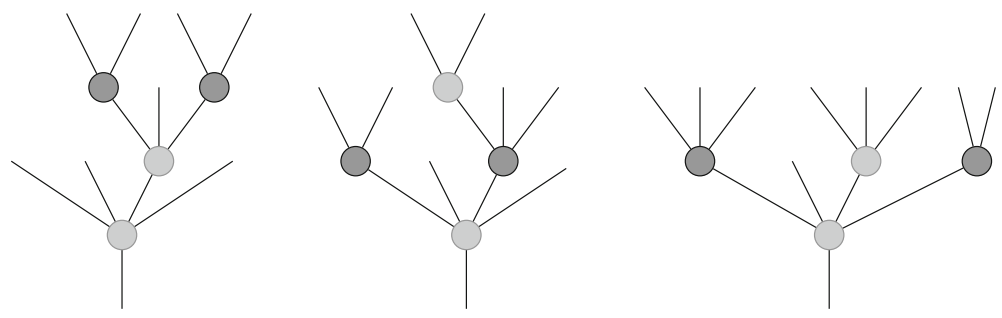

Finally, applying $\delta_{s} \delta_{t}$ will produce for us terms of the unique shape

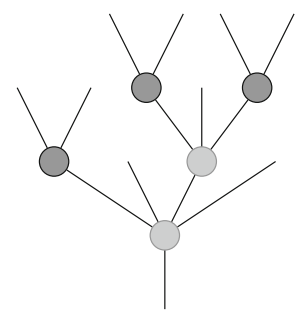

obtained by applying $\delta_{s}$ to a dark dot above a light one in "bouquet" produced by $\delta_{t}$; in this picture there is always at least one dark dot on the leaves of the lowest light dot, and at least two dark dots in the leaves of the highest light dot.

The fact that $\Delta$ is coassociative implies that the following shapes pair up to cancel:

- the second and third shapes in the first figure,

- the first shape in the first figure and the only shape in the third figure,

- the fourth shape in the first figure and the third shape in the second figure,

- The fourth shape and the fifth shape in the first figure with the first shape and the second shape in the second figure, respectively.

This gives what we wanted.

\section{Applications, examples and computations}

\subsection{Algebras with a tower of derivations}

Let us show how to use the methods of the last subsection to recover all the results in the article [6] of A. Das. As it is done there, consider the operad Das ${ }_{N}$ governing associative algebras with a tower of "higher order" derivations of height $N$. These consist of a vector space $V$ with an associative product, which we write $x_{1} x_{2}$, along with $N$ linear operators $\partial_{1}, \ldots, \partial_{N}: V \longrightarrow V$ such that

$$
\partial_{t}\left(x_{1} x_{2}\right)-\partial_{t} x_{1} x_{2}-x_{1} \partial_{t} x_{2}=\sum_{a+b=t} \partial_{a}\left(x_{1}\right) \partial_{b}\left(x_{2}\right), \quad \text { for } t \in\{1, \ldots, N\}
$$

Since it will make no difference in our computations, we will consider the case where we have an infinite tower of such operators, and write the corresponding operad Das. 
Naturally, Das contains a tower of of suboperads

$$
\text { Ass }=\operatorname{Das}_{0} \subseteq \operatorname{Das}_{1} \subseteq \cdots \subseteq \text { Das. }
$$

We now observe that this operad falls within the formalism we developed in Sect. 3.4. Indeed, let $C$ be the coalgebra with basis $\left\{\partial_{i}, i \in \mathbb{N}_{0}\right\}$ and coproduct

$$
\Delta\left(\partial_{n}\right)=\sum_{i+j=n} \partial_{i} \otimes \partial_{j},
$$

and let $H=T(C)$ as before. Then Ass $_{H}$ coincides exactly with Das, and if we restrict our construction to the subcoalgebra $C_{N}$ of $C$ with basis

$$
1=\partial_{0}, \partial_{1}, \ldots, \partial_{N}
$$

we obtain a suboperad of $\operatorname{Ass}_{H}$ that coincides with Das ${ }_{N}$.

To address the summand of the differential containing the perturbation, it is enough that we observe the iterated comultiplication of $C$ is such that for each $j \geq 2$,

$$
\Delta^{(j)}\left(\partial_{n}\right)=\sum_{|\vec{i}|=n} \partial_{i_{1}} \otimes \cdots \otimes \partial_{i_{j}}
$$

as the sum runs through all tuples $\vec{i}=\left(i_{1}, \ldots, i_{j}\right)$ with sum equal to $n$. It follows that to compute

$$
D_{j-1}\left(\partial_{n} \otimes \mu_{m}\right)
$$

we need only consider terms of the form

$$
\mu_{k_{0} \circ \vec{m}}\left(\partial_{1} \otimes \mu_{k_{1}}, \ldots, \partial_{j} \otimes \mu_{k_{j}}\right)
$$

where $\vec{k}$ is admissible for $m$. In other words, the terms appearing in $D\left(\partial_{n} \otimes \mu_{n}\right)$ are obtained by decorating an operation of $\mu_{k_{0}}$ by operations $\partial_{i_{j}} \otimes \mu_{k_{j}}$ so that $|\vec{a}|=n$ and the arities match, that is, $k_{0}+k_{1}+\cdots+k_{j}=n+j$.

Let us now fix an $\operatorname{Das}_{N}$-algebra $A$ and consider the corresponding model $\mathbb{M}\left(\operatorname{Das}_{N}\right)$ of $\operatorname{Das}_{N}$ obtained by keeping elements in $\mathbb{M}($ Das $)$ decorated only by labels in $\{1, \ldots, N\}$, and look at the deformation complex

$$
\operatorname{Def}(\mathrm{id}: A \longrightarrow A)=\operatorname{Der}\left(\mathbb{M}\left(\operatorname{Das}_{N}\right)(A), A\right)=\operatorname{hom}\left(q \operatorname{Das}^{i}(A), A\right)[1]
$$

Note that this is bigraded by arity and internal weight. Moreover, we can write generators of $q \operatorname{Das}^{i}(A)$ by decorated bar elements

$$
\left[x_{1}|\cdots| x_{n}\right]_{i}
$$


with the convention that for $i=0$ there is no decoration; these last generators correspond to a part of this space which equals $B A$. In general, such generators correspond to the arity $n$ cooperation

$$
\left(\partial_{i} \otimes \mu_{n}\right)\left(x_{1}, \ldots, x_{n}\right)
$$

and they are in bidegree $(n, i)$, where $i$ is the internal weight degree. In this way, we can specify a derivation by a collection $f=\left(f_{i, n}\right)$ of maps

$$
f_{i, n}: A^{\otimes n} \longrightarrow A, \text { for } i=0, \ldots, N
$$

in such a way that $f$ acts on a bar term of length $n$ decorated by $i$ by $f_{i, n}$. The differential is given by precomposition with $D: \mathbb{M}$ (Das) $\longrightarrow \mathbb{M}$ (Das) and the action on $A$. Since the projection $\mathbb{M}$ (Das) $\longrightarrow$ Das vanishes on non-zero homological degrees, the differential of some $f=\left(f_{m, n}\right)$ in bidegree $(m, n)$ contains the following terms:

$$
\begin{aligned}
(d f)^{\prime}\left[x_{1}|\cdots| x_{n}\right]_{m}= & \sum_{i+j=m} \partial_{i}\left(x_{1}\right) f\left[x_{2}|\cdots| x_{n}\right]_{j} \\
& +\sum_{i=1}^{i-1}(-1)^{i} f\left[x_{1}|\cdots| x_{i} x_{i+1}|\cdots| x_{n}\right]_{m} \\
& +(-1)^{n+1} \sum_{i+j=m} f\left[x_{1}|\cdots| x_{n-1}\right]_{i} \partial_{j}\left(x_{n}\right)
\end{aligned}
$$

coming from the non-Koszul higher terms of the form

$$
\left(1 \otimes \mu_{2}\right)\left(\partial_{i} \otimes 1, \partial_{j} \otimes \mu_{n-1}\right), \quad\left(1 \otimes \mu_{2}\right)\left(\partial_{i} \otimes \mu_{n-1}, \partial_{j} \otimes 1\right)
$$

and the Koszul terms

$$
\left(\partial_{m} \otimes \mu_{n}\right) \circ_{i}\left(1 \otimes \mu_{2}\right), \quad\left(1 \otimes \mu_{2}\right) \circ_{1}\left(\partial_{m} \otimes \mu_{n}\right), \quad\left(1 \otimes \mu_{2}\right) \circ_{2}\left(\partial_{m} \otimes \mu_{n}\right)
$$

in $D\left(\partial_{m} \otimes \mu_{n}\right)$. It is possible that $i=0$ or $j=0$ in the sums, and this gives the usual Ass $^{c}$ part of the differential. This is the "Hochschild" part of the differential the author defines in [6].

There are still a second part to the differential corresponding to the part of the differential of $\partial_{m} \otimes \mu_{n}$ where we have $\mu_{n}$ with differentials grafted at the leaves or at the root, this survives and gives the term

$$
\begin{aligned}
d f\left[x_{1}|\cdots| x_{n}\right]_{m}= & (-1)^{n} \sum_{i_{1}+\cdots+i_{k}=m} f\left[d_{i_{1}}\left(x_{1}\right)|\cdots| d_{i_{n}}\left(x_{n}\right)\right] \\
& -(-1)^{n} d_{m} f\left[x_{1}|\cdots| x_{n}\right] .
\end{aligned}
$$

The differential in the deformation complex is then the difference $d^{\prime}-d^{\prime \prime}$. 
The gradation of [6] corresponds the totalization of the bigradation that uses the external weight grading. Hence the entries $f_{0, n}, f_{1, n-1}, \ldots, f_{N, n-1}$ correspond to the linear functions $f, f_{1}, \ldots, f_{N}$ of [6], but note their differentials are still homogeneous with respect to the internal weight. In this way, the term

$$
C^{n}(A)=\operatorname{hom}\left(A^{\otimes n}, A\right) \oplus \operatorname{hom}\left(A^{\otimes(n-1)}, A\right)^{N}
$$

appearing in this paper is in total bidegree $n$ if we totalize the deformation complex using the bidegree given by external weight and arity.

Other variations. By considering homotopy associative algebras with derivations where the operators $\partial_{i} \otimes \mu_{n}$ vanish for $n \geq 2$, we obtain the definition of an $A_{\infty^{-}}$ algebra with higher derivations. In the same way, by considering homotopy associative algebras with higher order derivations where the operators $\mu_{n}$ vanish for $n \geq 2$, we obtain the definition of an associative algebra with a homotopy coherent system of higher order derivations.

\subsection{Lie group actions}

Let us consider a Lie group $G$ along with a left $\lambda: G \times M \longrightarrow M$ action on a differentiable manifold $M$, and as usual write $\mathfrak{g}$ for the Lie algebra of $G$. For each $p \in M$ we have a map

$$
\sigma_{p}: G \longrightarrow M
$$

and in particular we can define for each $X \in \mathfrak{g}$ the fundamental vector field $X^{\#}$ generated by $X$ by the requirement that for each $p \in M$ we have

$$
X_{p}^{\#}=\left.\frac{d}{d t}\right|_{t=0} \sigma_{p}(\exp (-t X)) .
$$

Proposition 4.1 The map $\xi: \mathfrak{g} \longrightarrow \mathfrak{X}(M)$ such that $X \longmapsto X^{\#}$ defines a $\mathfrak{g}$-algebra structure on the algebra $C^{\infty}(M)$ of smooth functions on $M$.

This proposition then gives us a natural geometrical source of examples of (commutative) associative algebras $A$ in left $\mathfrak{g}$-modules. These consist of "half" the amount of data defining a Lie-Reinhart pair $(\mathfrak{g}, A)$ - the algebraic avatar corresponding to Lie algebroids - in the sense we are not requiring $\mathfrak{g}$ to be a left $A$-module for which the following Leibniz rule

$$
[X, a Y]=X(a) Y+a[X, Y]
$$

holds for arbitrary $X, Y \in \mathfrak{g}$ and $a \in A$.

As an example, let us consider the Hopf algebra $H=\mathcal{U}(\mathfrak{g})$ where $\mathfrak{g}=\langle e, f, h\rangle$ is the Lie algebra of the Lie group $\operatorname{SL}(2, \mathbb{C})$ of complex matrices with determinant equal to 1 , so that 


$$
[h, e]=2 e, \quad[h, f]=-2 f, \quad[e, f]=h
$$

This bialgebra admits a PBW basis of the form $\left\{e^{i} f^{j} h^{k}: i, j, k \in \mathbb{N}\right\}$ and a Koszul model with generators the exterior coalgebra $H^{i}=S^{c}\left(s \mathfrak{g}^{*}\right)$ on the shift of the dual $\mathfrak{g}^{*}=\left\langle e^{*}, f^{*}, h^{*}\right\rangle$ which is nothing but the Chevalley-Eilenberg complex $\mathrm{e}^{*}(\mathfrak{g})$ of $\mathfrak{g}$. As such, it is finite dimensional (of dimension 8) and we obtain a (non-minimal) model of $\operatorname{Ass}_{H}$ with generators

$$
\mathcal{C}^{*}(\mathfrak{g}) \otimes \text { Ass }^{i}
$$

In this case, the non-minimality is due to the appereance of linear terms in the defining relations of $\mathcal{U}(\mathfrak{g})$. The spectral sequence above takes the following form:

Theorem 4.2 Let $A$ be an associative $\mathfrak{g}$-algebra and let $M$ be an operadic A-module. There is a convergent spectral sequence

$$
E_{2}^{p, q}=H^{p}\left(\mathfrak{g}, H^{q}(A, M)\right) \stackrel{p}{\Longrightarrow} H_{\mathfrak{g}}^{p+q}(A, M),
$$

where $H^{*}(A, M)$ is the Hochschild cohomology of $A$ with values in $M, H^{*}(\mathfrak{g},-)$ is the Lie algebra cohomology functor of $\mathfrak{g}$, and $H_{\mathfrak{g}}^{*}(A, M)$ is the operadic cohomology of $A$ with values in $M$.

It is interesting to compare this spectral sequence with the spectral sequence of [23]. In that article the authors start with a Lie-Rinehart pair $(\mathfrak{g}, S)$ [35]. These pairs are known [21] to admit a universal enveloping algebra $U=U(\mathfrak{g}, S)$ for which $(\mathfrak{g}, S)$ modules are exactly the left $U$-modules. Many associative algebras of interest, such as the Weyl algebras and algebras of differentials forms tangent to a free hyperplane arrangement, for example, arise as such universal envelopes for suitable choices of $\mathfrak{g}$ and $S$.

One of the main results in that paper (Theorem B) is the following, where $H_{S}^{*}(\mathfrak{g},-)$ is the Lie-Rinehart cohomology functor associated to the Lie-Rinehart pair $(\mathfrak{g}, S)$, given by the functor $\operatorname{Ext}_{U}^{*}(S,-)$.

Theorem Let $(\mathfrak{g}, S)$ be a Lie-Rinehart pair such that $\mathfrak{g}$ is an S-projective module and let $M$ be an $U$-bimodule. There exist a $U$-module structure on $H^{*}(S, M)$ and a first-quadrant spectral sequence converging to $H^{*}(U, M)$ with second page

$$
E_{2}^{p, q}=H_{S}^{p}\left(\mathfrak{g}, H^{q}(S, M)\right) \stackrel{p}{\Longrightarrow} H^{p+q}(U, M) .
$$

\subsection{Unstable algebras over the Steenrod algebra}

In this section, $\mathbb{k}$ is the field of two elements. Let $\mathcal{A}$ be the mod-2 Steenrod algebra. It is generated by the Steenrod squares

$$
1=\mathrm{Sq}^{0}, \mathrm{Sq}^{1}, \mathrm{Sq}^{2}, \ldots
$$


subject to the Adem relations and is, in fact, a cocommutative Hopf algebra. These relations say that for each $i, j \in \mathbb{N}$ such that $i<2 j$, we have that

$$
\mathrm{Sq}^{i} \mathrm{Sq}^{j}=\left(\begin{array}{c}
j-1 \\
i
\end{array}\right) \mathrm{Sq}^{i+j}+\sum_{k \geq 1}\left(\begin{array}{c}
j-k-1 \\
i-2 k
\end{array}\right) \mathrm{Sq}^{i+j-k} \mathrm{Sq}^{k}
$$

Its comultiplication is such that for each $n \in \mathbb{N}$,

$$
\Delta\left(\mathrm{Sq}^{n}\right)=\sum_{i+j=n} \mathrm{Sq}^{i} \otimes \mathrm{Sq}^{j}
$$

Note that, depending on the parity of the binomial coefficient $\left(\begin{array}{c}j-1 \\ i\end{array}\right)$, there may appear a linear term to the right, which makes this presentation of $H$ into a linear-quadratic one. However, this is enough to obtain, for example, the following well known result giving a linear basis for $\mathcal{A}$, usually known as the Cartan-Serre basis:

Theorem For each tuple $I=\left(i_{1}, \ldots, i_{n}\right)$, consider the monomial $\mathrm{Sq}^{I}=\mathrm{Sq}^{i_{1}} \cdots \mathrm{Sq}^{i_{n}}$. Then the set of monomials $B=\left\{\mathrm{Sq}^{I}: i_{j} \geq 2 i_{j+1}\right.$ for each $\left.j\right\}$ forms a linear basis of $\mathcal{A}$.

The dual $\mathcal{A}^{*}$ of $\mathcal{A}$ was determined in a well known paper [29] of Milnor:

Theorem (J. Milnor) The dual Hopf algebra $\mathcal{A}^{*}$ is a free graded commutative algebra on generators $\xi_{1}, \xi_{2}, \ldots$ where for each $i \in \mathbb{N}$ the element $\xi_{i}$ is of degree $2^{i}-1$ and corresponds to the dual of the monomial $\mathrm{Sq}^{2}{ }^{i-1} \ldots \mathrm{Sq}^{2} \mathrm{Sq}^{1}$ in the Cartan-Serre basis. Its comultiplication is given by the formula

$$
\Delta\left(\xi_{k}\right)=\sum_{i+j=k} \xi_{i}^{2^{j}} \otimes \xi_{j} \text { for each } k \in \mathbb{N} .
$$

The underlying algebra of $\mathcal{A}$ is inhomogeneous Koszul -in fact, the study of this quadratic algebra is one of the reasons Priddy [34] introduced the notion of a Koszul algebra in the first place. Note that Priddy calls "homogeneous Koszul algebras" what are now usually called Koszul algebras, and calls "Koszul algebras" what are now called inhomogenous Koszul algebras. One of the main results of [34] is the following:

Theorem (Priddy) The Steenrod algebra $\mathcal{A}$ is inhomogenous Koszul. Moreover, the dg algebra $\mathcal{A}^{!}$is isomorphic to the opposite of the $\Lambda$ algebra constructed in [3], which computes the Yoneda algebra $\operatorname{Ext}_{\mathcal{A}}(\mathbb{k}, \mathbb{k})$.

The dg algebra $\Lambda$ is generated by symbols $\lambda_{n}$ for $n \in \mathbb{N}_{0}$ of cohomological degree 1 and weight $n+1$ subject to the family of quadratic relations for $m-1, n \in \mathbb{N}$

$$
\sum_{i+j=n}\left(\begin{array}{c}
i+j \\
i
\end{array}\right) \lambda_{i-1+m} \lambda_{j-1+2 m}=0
$$


and differential so that for each $n \in \mathbb{N}$ we have

$$
d \lambda_{n-1}=\sum_{i+j=n}\left(\begin{array}{c}
i+j \\
i
\end{array}\right) \lambda_{i-1} \lambda_{j-1} .
$$

Theorem (Bousfield et al.) The algebra $\Lambda$ is equal to the $E_{1}$ page appearing in the Adams spectral sequence converging to the 2-component of the stable homotopy groups of spheres. In particular, there is a bigraded isomorphism of algebras

$$
H(\Lambda) \longrightarrow \operatorname{Ext}_{\mathcal{A}}(\mathbb{k}, \mathbb{k}) .
$$

The discussion above together with our main results show the following:

Theorem 4.3 There is a model of the operad Ass $\mathcal{A}_{\mathcal{A}}$ controlling associative algebras in $\mathcal{A}$-modules with generators $s^{-1} \mathcal{A}^{i} \otimes$ Ass $^{i}$ where $\mathcal{A}^{i}$ is the Koszul dual dg coalgebra of the inhomogeneous Koszul Steenrod algebra $\mathcal{A}$.

It is important to remark that the "correct" Koszul duality theory to consider when dealing with topological spaces is not the one provided by the self dual pair (Ass, Ass) but rather by the pair (Com, Lie); see [2]. Hence, one should put efforts in considering the symmetric operad $\operatorname{Com}_{\mathcal{A}}$ instead, controlling commutative $\mathcal{A}$-algebras. Doing this introduces a complication: in positive characteristic, the homotopy theory of commutative algebras - and hence, of commutative $\mathcal{A}$-algebras - requires the use of simplicial as opposed to dg methods; see, for example, the wonderful monograph [18].

It is also useful to remark that there are other presentations of $\mathcal{A}$ in the literature which, instead of being linear-quadratic, are "perturbative". By this, we mean they are obtained from a bona-fide quadratic presentation (of a different algebra) by adding higher terms, which may be cubic, cuartic, and so forth. For example, in [42], Wall considers the generators $S=\left\{\mathrm{Sq}^{1}, \mathrm{Sq}^{2}, \mathrm{Sq}^{4}, \ldots\right\}$. The main theorem in that article is the following.

Theorem (Wall) The Steenrod algebra $\mathcal{A}$ is isomorphic to the quotient of the free associative algebra generated by $S$ subject to relations of the form

$$
\begin{array}{rlrl}
\mathrm{Sq}^{2^{i}} \mathrm{Sq}^{2^{i}}+\mathrm{Sq}^{2^{i-1}}\left[\mathrm{Sq}^{2^{i}}, \mathrm{Sq}^{2^{i-1}}\right] & \in \mathcal{A}(i-1) & \text { for each } i & \geq 0 \\
{\left[\mathrm{Sq}^{2}, \mathrm{Sq}^{2^{j}}\right]} & \in \mathcal{A}(i-1) & \text { for each } i-j \geq 2
\end{array}
$$

where $\mathcal{A}(i)$ is the subalgebra generated by $\mathrm{Sq}^{1}, \mathrm{Sq}^{2}, \ldots, \mathrm{Sq}^{2}$.

In this way, Wall exhibits $\mathcal{A}$ as a pertubation of an algebra which almost looks like an exterior algebra-the relations missing are of the form $\left[\mathrm{Sq}^{2^{i}}, \mathrm{Sq}^{2^{i-1}}\right]$ - and uses this presentation to successfully solve a problem Toda posed in [41]. In fact, in unpublished work Walker has determined these relations explicitly in terms of the 
antipode $\chi$ of $\mathcal{A}$, see Theorem 4.18 in [43]. We point out that a result of Straffin [39] shows that

$$
\chi\left(\mathrm{Sq}^{2^{i}}\right)=\mathrm{Sq}^{2^{i}}+\mathrm{Sq}^{2^{i-1}} \chi\left(\mathrm{Sq}^{2^{i-1}}\right)
$$

For the primitive $\mathrm{Sq}^{1}$ we of course have $\chi\left(\mathrm{Sq}^{1}\right)=\mathrm{Sq}^{1}$, so this and the recursive formula above gives an efficient way of computing the antipode for the generating set of Wall. For example, since $\mathrm{Sq}^{1} \mathrm{Sq}^{1}=0$ and $\mathrm{Sq}^{2} \mathrm{Sq}^{2}=\mathrm{Sq}^{1} \mathrm{Sq}^{2} \mathrm{Sq}^{1}$, we have that

$$
\chi\left(\mathrm{Sq}^{2}\right)=\mathrm{Sq}^{2}, \quad \chi\left(\mathrm{Sq}^{4}\right)=\mathrm{Sq}^{4}+\mathrm{Sq}^{1} \mathrm{Sq}^{2} \mathrm{Sq}^{1}
$$

Theorem (Walker) The closed forms of the Wall relations are given by

$$
\begin{array}{rrr}
\mathrm{Sq}^{2^{i}} \mathrm{Sq}^{2^{i}}=\mathrm{Sq}^{2^{i-1}}\left[\chi\left(\mathrm{Sq}^{2^{i}}\right), \mathrm{Sq}^{2^{i-1}}\right] & \text { for } i \geq 0, \\
{\left[\mathrm{Sq}^{2^{i}}, \chi\left(\mathrm{Sq}^{2^{j}}\right)\right]=\chi\left(\mathrm{Sq}^{2^{j+1}}\right) \mathrm{Sq}^{2^{i}-2^{j}}} & \text { for } i-j \geq 2,
\end{array}
$$

where $\chi$ is the antipode of $\mathcal{A}$.

The way $\mathcal{A}$ appears naturally in homotopy theory is as follows. For any topological space $X$ the cohomology groups $H^{*}(X, \mathbb{Z} / 2)$ form a (commutative) associative algebra. Moreover, by virtue of the Cartan relations, that take up the form

$$
\mathrm{Sq}^{n}(x \smile y)=\sum_{i+j=n} \mathrm{Sq}^{i}(x) \smile \mathrm{Sq}^{j}(y),
$$

the coproduct of $\mathcal{A}$ is compatible with the algebra structure of $H^{*}(X, \mathbb{Z} / 2)$. In other words, we have the well known observation pioneered by Milnor [29]:

Theorem (Milnor) For each topological space $X$ the cohomology groups $H^{*}(X, \mathbb{Z} / 2)$ form a commutative associative algebra in left $\mathcal{A}$-modules.

These $\mathcal{A}$-algebras, which arise as cohomology algebras of spaces, are unstable $\mathcal{A}$-algebras, in that they satisfy the additional instability condition that

$$
\mathrm{Sq}^{i}(x)= \begin{cases}0 & \text { for } i>|x| \\ x^{2} & \text { for } i=|x|\end{cases}
$$

To illustrate, one has the classical example of infinite projective space $\mathbb{R} P^{\infty}$, for which $A=H^{*}\left(\mathbb{R} P^{\infty}, \mathbb{Z} / 2\right)$ is a polynomial algebra $\mathbb{k}[x]$ with $|x|=1$. The action of $\mathcal{A}$ is given by

$$
\operatorname{Sq}^{i}\left(x^{n}\right)=\left(\begin{array}{l}
n \\
i
\end{array}\right) x^{n+i} \quad \text { for each } n, i \in \mathbb{N} .
$$

To give an example of Corollary 3.4, we will content ourselves with applying it to the sub-Hopf algebra $\mathcal{A}(2)$ of $\mathcal{A}$ generated by $\mathrm{Sq}^{1}=x, \mathrm{Sq}^{2}=y$ and $\mathrm{Sq}^{4}=z$. Following the method of Wall or explicitly using the relations of Walker, one can check that: 
Proposition 4.4 The Hopf algebra $\mathcal{A}(2)$ has dimension 64 and admits a presentation with defining relations those of $\mathcal{A}(1)$ and the two relations $z^{2}=y[z, y]$ and $[x, z]=$ yxy. The set $G(2)$ consisting of the following polynomials forms a Gröbner basis for $\mathcal{A}(2)$ :

\begin{tabular}{ll}
\hline Leading term & Corresponding relation \\
\hline$x^{2}$ & $x^{2}$ \\
$y^{2}$ & $y^{2}+x y x$ \\
$z^{2}$ & $z^{2}+y[z, y]$ \\
$z x$ & {$[z, x]+y x y$} \\
$y x y x$ & $(y x)^{2}+(x y)^{2}$ \\
$z y z y$ & $(z y)^{2}+(y z)^{2}+(x y)^{2} z y+x z y x z$ \\
$z y x y$ & {$[z, y x y]+[x, y z y]$} \\
$z y x z y x$ & $(z y x)^{2}+(x z y)^{2}+(y x z)^{2}+(x y x)(z y z)$ \\
\hline
\end{tabular}

Proof One can compute the extra relations using Buchberger's algorithm, and then check that it gives exactly 64 normal forms, so there cannot be more elements in the Gröbner basis.

Corollary 4.5 The set $G(2) \cup R_{\mu} \cup R_{\lambda}$ is a finite Gröbner basis for the operad $\operatorname{Ass}_{\mathcal{A}(2)}$.

Note that the results we obtained here about Gröbner bases work equally well for the operad $\operatorname{Com}_{\mathcal{A}}$.

Acknowledgements The author thanks Vladimir Dotsenko for his suggestion to extend our original results, which mostly centered in applying the ideas of Sect. 3.4 to explain the work done in [6], to a more general setting.

Funding Open Access funding enabled and organized by Projekt DEAL.

Open Access This article is licensed under a Creative Commons Attribution 4.0 International License, which permits use, sharing, adaptation, distribution and reproduction in any medium or format, as long as you give appropriate credit to the original author(s) and the source, provide a link to the Creative Commons licence, and indicate if changes were made. The images or other third party material in this article are included in the article's Creative Commons licence, unless indicated otherwise in a credit line to the material. If material is not included in the article's Creative Commons licence and your intended use is not permitted by statutory regulation or exceeds the permitted use, you will need to obtain permission directly from the copyright holder. To view a copy of this licence, visit http://creativecommons.org/licenses/by/4.0/.

\section{References}

1. Beck, J.: Distributive Laws Seminar on Triples and Categorical Homology Theory (ETH, Zürich, 1966/67), pp. 119-140. Springer, Berlin (1969)

2. Berglund, A.: Koszul spaces. Trans. Am. Math. Soc. 366(9), 4551-4569 (2014). https://doi.org/10. 1090/S0002-9947-2014-05935-7 
3. Bousfield, A.K., Curtis, E.B., Kan, D.M., Quillen, D.G., Rector, D.L., Schlesinger, J.W.: The mod - $p$ lower central series and the Adams spectral sequence. Topology 5, 331-342 (1966). https://doi.org/ 10.1016/0040-9383(66)90024-3

4. Cisinski, D.-C., Moerdijk, I.: Dendroidal sets and simplicial operads. J. Topol. 6(3), 705-756 (2013). https://doi.org/10.1112/jtopol/jtt006

5. Das, A.: Cohomology and deformations of dendriform coalgebras (2019)

6. Das, A.: Extensions and deformations of algebras with higher derivations (2020)

7. Das, A.: Leibniz algebras with derivations (2020)

8. Das, A., Mandal, A.: Extensions, deformation and categorification of AssDer pairs (2020)

9. Dolgushev, V., Willwacher, T.: Operadic twisting-with an application to Deligne's conjecture. J. Pure Appl. Algebra 219(5), 1349-1428 (2015)

10. Dotsenko, V.: Word operads and admissible orderings. Appl. Categ. Struct. 28(4), 595-600 (2020). https://doi.org/10.1007/s10485-020-09591-0

11. Dotsenko, V., Foissy, L.: Operads of enriched pre-lie algebras and freeness theorems (2020)

12. Dotsenko, V., Shadrin, S., Vallette, B.: The twisting procedure. https://hal.archives-ouvertes.fr/hal02378802 (2019) (New format (short monography), 93 pages, minor corrections, submitted version)

13. Dotsenko, V., Vallette, B.: Higher Koszul duality for associative algebras. Glasg. Math. J. 55(A), 55-74 (2013). https://doi.org/10.1017/S0017089513000505

14. Drummond-Cole, G.C., Vallette, B.: The minimal model for the Batalin-Vilkovisky operad. Sel. Math. (N.S.) 19(1), 1-47 (2013). https://doi.org/10.1007/s00029-012-0098-y

15. Fresse, B.: On the homotopy of simplicial algebras over an operad. Trans. Am. Math. Soc. 352(9), 4113-4141 (2000). https://doi.org/10.1090/S0002-9947-99-02489-7

16. Gálvez-Carrillo, I., Tonks, A., Vallette, B.: Homotopy Batalin-Vilkovisky algebras. J. Noncommut. Geom. 6(3), 539-602 (2012). https://doi.org/10.4171/JNCG/99

17. Ginot, G.: Homologie et modèle minimal des algèbres de Gerstenhaber. Ann. Math. Blaise Pascal 11(1), 95-127 (2004). http://ambp.cedram.org/item?id=AMBP_2004_11_1_95_0

18. Goerss, P., Turner, J.: Homotopy theory of simplicial abelian Hopf algebras. J. Pure Appl. Algebra 135(2), 167-206 (1999). https://doi.org/10.1016/S0022-4049(97)00142-4

19. Hazewinkel, Michiel: The primitives of the hopf algebra of noncommutative symmetric functions. São Paulo J. Math. Sci. 1(2), 175 (2007-212). https://doi.org/10.11606/issn.2316-9028.v1i2p175-202

20. He, J.-W., Lu, D.-M.: Higher koszul algebras and a-infinity algebras. J. Algebra 293(2), 335-362 (2005)

21. Huebschmann, J.: Poisson cohomology and quantization. J. Reine Angew. Math. 408, 57-113 (1990). https://doi.org/10.1515/crll.1990.408.57

22. Khoroshkin, A., Tamaroff, P.: Derived poincaré-birkhoff-witt theorems (with an appendix by vladimir dotsenko) (2020)

23. Kordon, F., Lambre, T.: Lie-Rinehart and Hochschild cohomology for algebras of differential operators. J. Pure Appl. Algebra 225(1), 106456 (2021). https://doi.org/10.1016/j.jpaa.2020.106456

24. Kumar, N.: A survey on Koszul algebras and Koszul duality. In: Ambily, A.A., Hazrat, R., Sury, B. (eds.) Leavitt Path Algebras and Classical k-Theory, pp. 157-176. Springer Singapore, Singapore (2020)

25. Lada, T., Markl, M.: Strongly homotopy lie algebras. Commun. Algebra 23(6), 2147-2161 (1995). https://doi.org/10.1080/00927879508825335

26. Loday, J.-L., Vallette, B.: Algebraic Operads, Grundlehren der Mathematischen Wissenschaften [Fundamental Principles of Mathematical Sciences], vol. 346. Springer, Heidelberg (2012)

27. Markl, M.: Distributive laws and Koszulness, Ann. Inst. Fourier (Grenoble) 46(2), 307-323 (1996). http://www.numdam.org/item?id=AIF_1996_46_2_307_0

28. Merkulov, S., Vallette, B.: Deformation theory of representations of prop(erad)s. I. J. Reine Angew. Math. 634, 51-106 (2009). https://doi.org/10.1515/CRELLE.2009.069

29. Milnor, J.: The Steenrod algebra and its dual. Ann. Math. 2(67), 150-171 (1958). https://doi.org/10. 2307/1969932

30. Milnor, J.W., Moore, J.C.: On the structure of Hopf algebras. Ann. Math. 2(81), 211-264 (1965). https://doi.org/10.2307/1970615

31. Moerdijk, I., Toën, B.: Simplicial methods for operads and algebraic geometry. In: Carles, C., Joachim, K. (eds.) Advanced Courses in Mathematics. CRM Barcelona. Springer Basel AG, Basel (2010). https:// doi.org/10.1007/978-3-0348-0052-5 
32. Novelli, J.-C., Thibon, J.-Y.: Noncommutative symmetric functions and lagrange inversion. Adv. Appl. Math. 40(1), 8-35 (2008-01). https://doi.org/10.1016/j.aam.2007.05.005

33. Polishchuk, A., Positselski, L.: Quadratic Algebras. University Lecture Series, p. 37. American Mathematical Society, Providence (2005). https://doi.org/10.1090/ulect/037

34. Priddy, S.B.: Koszul resolutions. Trans. Am. Math. Soc. 152, 39-60 (1970). https://doi.org/10.2307/ 1995637

35. Rinehart, G.S.: Differential forms on general commutative algebras. Trans. Am. Math. Soc. 108, 195222 (1963). https://doi.org/10.2307/1993603

36. Singer, W.M.: On the minimality of Anick's resolution, with application to an algebra of cohomology operations. J. Algebra 179(3), 918-929 (1996). https://doi.org/10.1006/jabr.1996.0044

37. Stasheff, J.D.: Homotopy associativity of $H$-spaces I, II. Trans. Am. Math. Soc. 108, 275-292 (1963)

38. Stasheff, J.D.: Homotopy associativity of $H$-spaces I, II. Trans. Am. Math. Soc. 108, 293-312 (1963). https://doi.org/10.1090/s0002-9947-1963-0158400-5

39. Straffin, P.D., Jr.: Identities for conjugation in the Steenrod algebra. Proc. Am. Math. Soc. 49, 253-255 (1975). https://doi.org/10.2307/2039825

40. Tamarkin, D., Tsygan, B.: Noncommutative differential calculus, homotopy BV algebras and formality conjectures. Methods Funct. Anal. Topol. 6(2), 85-100 (2000)

41. Toda, H.: On exact sequences in Steenrod algebra mod 2. Mem. Coll. Sci. Univ. Kyoto Ser. A. Math. 31, 33-64 (1958). https://doi.org/10.1215/kjm/1250776948

42. Wall, C.T.C.: Generators and relations for the Steenrod algebra. Ann. Math. 2(72), 429-444 (1960). https://doi.org/10.2307/1970225

43. Wood, R.M.W.: Problems in the Steenrod algebra. Bull. Lond. Math. Soc. 30(5), 449-517 (1998). https://doi.org/10.1112/S002460939800486X

Publisher's Note Springer Nature remains neutral with regard to jurisdictional claims in published maps and institutional affiliations. 